\title{
Deep sequencing and SNP array analyses of pediatric T-cell acute lymphoblastic leukemia reveal NOTCH1 mutations in minor subclones and a high incidence of uniparental isodisomies affecting CDKN2A
}

Kristina Karrman ${ }^{1,2^{*}}$, Anders Castor ${ }^{3}$, Mikael Behrendtz $^{4}$, Erik Forestier ${ }^{5}$, Linda Olsson ${ }^{2}$, Mats Ehinger ${ }^{6}$, Andrea Biloglav', Thoas Fioretos ${ }^{1,2}$, Kajsa Paulsson ${ }^{2}$ and Bertil Johansson ${ }^{1,2}$

\begin{abstract}
Background: Pediatric T-cell acute lymphoblastic leukemia (T-ALL) is a genetically heterogeneous disease that arises in a multistep fashion through acquisition of several genetic aberrations, subsequently giving rise to a malignant, clonal expansion of T-lymphoblasts. The aim of the present study was to identify additional as well as cooperative genetic events in T-ALL.

Methods: A population-based pediatric T-ALL series comprising 47 cases was investigated by SNP array and deep sequencing analyses of 75 genes, in order to ascertain pathogenetically pertinent aberrations and to identify cooperative events.
\end{abstract}

Results: The majority (92\%) of cases harbored copy number aberrations/uniparental isodisomies (UPIDs), with a median of three changes (range 0-11) per case. The genes recurrently deleted comprised CDKN2A, CDKN2B, LEF1, PTEN, RBI, and STIL. No case had a whole chromosome UPID; in fact, literature data show that this is a rare phenomenon in T-ALL. However, segmental UPIDs (sUPIDs) were seen in $42 \%$ of our cases, with most being sUPID9p that always were associated with homozygous CDKN2A deletions, with a heterozygous deletion occurring prior to the sUPID9p in all instances. Among the 75 genes sequenced, 14 (19\%) were mutated in 28 (72\%) of 39 analyzed cases. The genes targeted are involved in signaling transduction, epigenetic regulation, and transcription. In some cases, NOTCH1 mutations were seen in minor subclones and lost at relapse; thus, such mutations can be secondary events.

Conclusions: Deep sequencing and SNP array analyses of T-ALL revealed lack of WUPIDs, a high proportion of sUPID9p targeting CDKN2A, NOTCH1 mutations in subclones, and recurrent mutations of genes involved in signaling transduction, epigenetic regulation, and transcription.

Keywords: T-ALL, Pediatric, Genetic characterization, SNP array, Large-scale sequencing

\footnotetext{
* Correspondence: Kristina.Karrman@med.lu.se

'Department of Clinical Genetics, University and Regional Laboratories,

Region Skåne, SE-221 85 Lund, Sweden

2Division of Clinical Genetics, Department of Laboratory Medicine, Lund

University, Lund, Sweden

Full list of author information is available at the end of the article
} 


\section{Background}

T-cell acute lymphoblastic leukemia (T-ALL) arises through a stepwise acquisition of genetic aberrations that occasionally are important for proper diagnosis and prognostication as well as for therapeutic decisions [1]. Cytogenetically, approximately half of T-ALL cases display clonal chromosome aberrations, with the cytogenetic and oncogenic hallmark of T-ALL being the presence of translocations leading to illegitimate rearrangements of the T-cell receptor (TCR) loci (mainly $T R A / T R D$ at $14 \mathrm{q} 11$ and TRB at $7 \mathrm{q} 34$ ). Genes targeted, and hence deregulated, by TCR rearrangements most often code for transcription factors or proteins involved in transcriptional complexes, such as the HOXA (7p15.2), LMO1 (11p15.4), LMO2 (11p13), TAL1 (1p33),
TLX1 (10q24.31), and TLX3 (5q35.1) genes [2]. However, additional abnormalities are required for overt leukemia. Typical examples include NOTCH1 (9q34.3) and FBXW7 (4q31.3) mutations and CDKN2A (9p21.3) deletions, which are present in the vast majority of T-ALL cases $[3,4]$. In addition, recent array and sequence analyses have implicated deletions and/or mutations in numerous other genes, with some of the most prevalent being ETV6 (12p13.2), EZH2 (7q36.1), IL7R (5p13.2), LEF1 (4q25), NRAS (1p13.2), PHF6 (Xq26.2), PTEN (10q23.31), RUNX1 (21q22.12), and WT1 (11p13) [5]. Here, we have used single nucleotide polymorphism (SNP) array and deep sequencing analyses of 75 selected candidate genes to characterize genetic aberrations in a consecutive series of pediatric T-ALL.

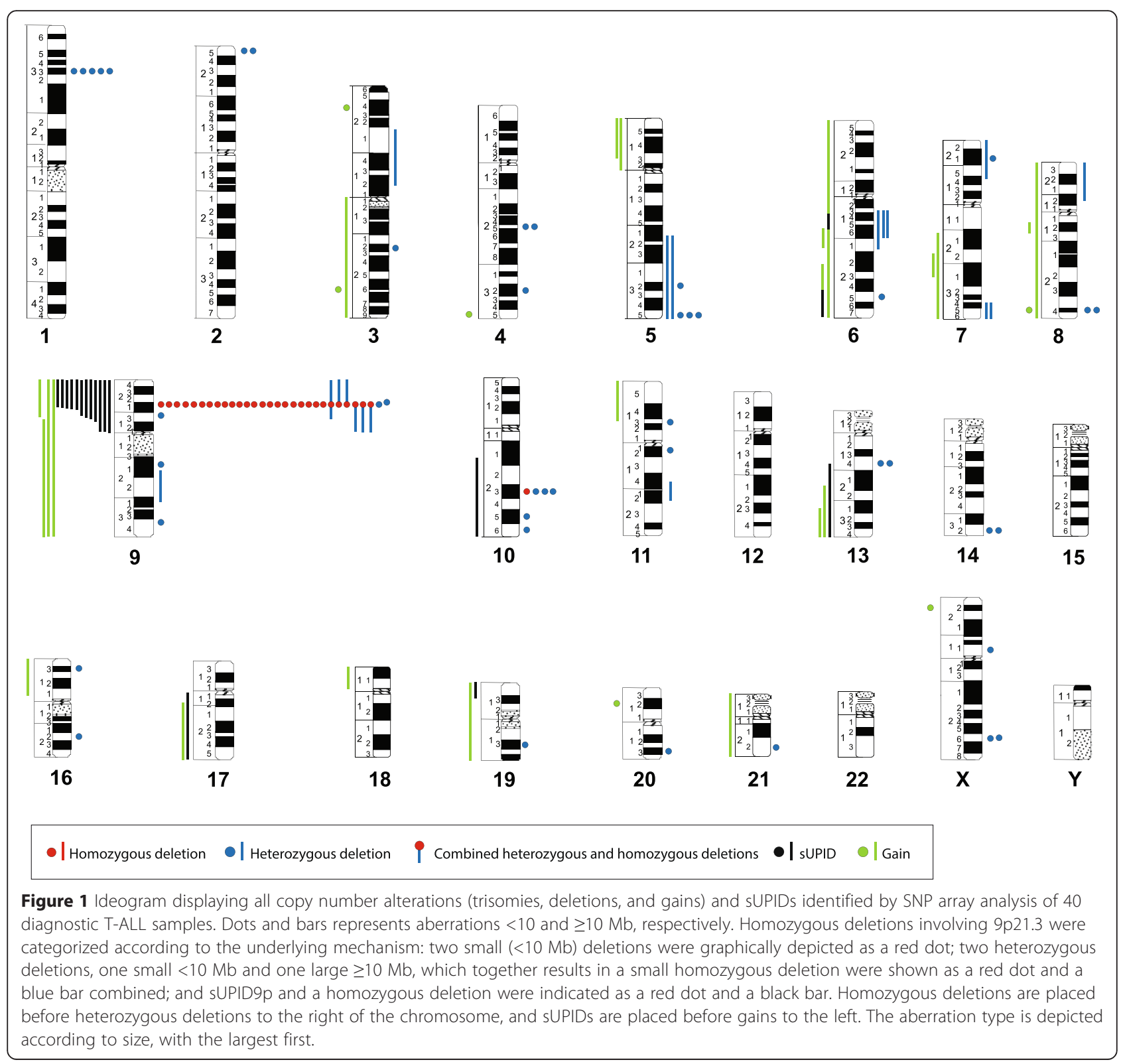




\section{Results}

\section{SNP array findings}

All aberrations found by SNP array analysis are listed in Additional file 1: Table S1 (using cytogenetic nomenclature) and Additional file 2: Table S2 (with detailed positions derived from the GRCh37 genome build), and are summarized graphically in Figure 1. Forty cases could be successfully analyzed. The vast majority (37/40; 92\%) harbored copy number abnormalities (CNAs), i.e., trisomies, deletions, or gains, and/or segmental uniparental isodisomies (sUPIDs). No monosomies or whole chromosome UPIDs (wUPIDs) were detected. In 37 of the investigated cases, karyotypic data were also available. The SNP array analyses yielded novel information in 35 (95\%) of these. In addition, the SNP arrays could better define the breakpoints and/or clarify ambiguous unbalanced changes detected by G-banding analysis in 13 (35\%) cases (Additional file 1: Table S1). All abnormalities seen by chromosome banding analysis leading to net gain or loss of genetic material were confirmed by SNP array analysis.

The analyses revealed 128 CNAs, of which 88 (69\%) were $<10 \mathrm{Mb}$ and 40 (31\%) were $\geq 10 \mathrm{Mb}$. The median number of CNAs per case was 3 (mean 3.3, range 0-10). Deletions were more common (100/128; 78\%) than gains $(28 / 128 ; 22 \%)$; the latter comprised trisomies $(n=6)$ and duplications $(n=22)$. In addition, 18 sUPIDs were found, all of which $\geq 10 \mathrm{Mb}$. Thus, a total of 146 abnormalities (CNAs and UPDs combined) were identified, with a median number of 3 (range 0-11) aberrations per case (Figure 1 and Additional file 2: Table S2).

\section{Large ( $\geq 10 \mathrm{Mb})$ genomic imbalances}

The only trisomies identified involved chromosomes 6 , $8,9,19$, and 21 (Figure 1). The smallest regions of overlap of recurrent large duplications were 5 p13.1-pter, 7q22.1-31.2, and 13q31.1-qter, whereas the smallest regions of overlap of recurrent large deletions were 5q21.3-qter, 6q14-16, 7q34-qter (within the TRB locus), 9p11-21.3, and 9p21.2-pter (Figure 1 and Additional file 1: Table S1).

\section{Small $(<10 \mathrm{Mb})$ genomic imbalances}

Six genes were recurrently targeted by deletions: 1 ) CDKN2A in 29/40 (72\%) cases, of which 28/29 (97\%) were homozygous deletions (either two separate deletions or one deletion in combination with sUPID9p); 2) CDKN2B (9p21.3) in 25/40 (62\%), all of which also involved CDKN2A; 3) STIL (1p33) in 5/40 (12\%), all of which were verified by interphase fluorescence in situ hybridization (FISH) analysis using the SIL-TAL1 subdeletion signal FISH probe (Dako, Glostrup, Denmark); 4) PTEN in $3 / 40$ (8\%); 5) LEF1 in $2 / 40$ (5\%); and 6) RB1 (13q14.2) in 2/40 (5\%). Deletions of PHF6 and upstream elements of $L M O 2$ were detected in one case each
(Figure 1). In four cases (\#31, 34, 36, and 37), no deletions involving the TCR genes, representing somatic recombination, were detected; however, the finding of other SNP-identified aberrations and/or high levels of mutated alleles, as ascertained by deep sequencing, in these cases proved the presence of leukemic cells in the investigated samples (Additional file 1: Table S1).

\section{sUPIDs}

Of the 18 sUPIDs identified in 17 (42\%) cases, only 9p was recurrently targeted (12 cases), including CDKN2A and $C D K N 2 B$ in all instances with the minimal overlap spanning 9p21.3-pter. All but one of the sUPIDs were terminal (Figure 1, Additional file 1: Table S1, and Additional file 2: Table S2). All sUPID9p were associated with homozygous $C D K N 2 A$ deletions; $C D K N 2 B$ was homozygously deleted in eight $(67 \%)$ of the 12 cases.

\section{Gene mutations}

All 75 genes targeted in the 39 cases analyzed could be successfully deep sequenced (2.1-3.2 million reads/sample). The mean target coverage varied between 268 times and 465 times per sample, with $98.3 \%-99.4 \%$ of the targets having at least 10 times coverage. Conversely, targets with no coverage ranged from $0.2 \%$ to $0.5 \%$ per sample.

A total of 46 mutations were detected among the 39 investigated cases at the time of diagnosis; the median was 1 mutation per sample (range 0-4) (Additional file 1: Table S1 and Additional file 3: Table S3). Mutations were identified in 28 (72\%) of the samples. All mutations were heterozygous, except for one case with a hemizygous mutation of the PHF6 gene at Xq26.2. Of the 46 mutations observed, 35 (76\%) were missense, six (13\%) indels, and five (11\%) were nonsense.

Details on all mutations are given in Additional file 3: Table S3. Of the 75 genes sequenced, 14 (19\%) were shown to be mutated at least once: $B C L 11 B$ at $14 \mathrm{q} 32.2$ $(n=1), C R E B B P$ at $16 \mathrm{p} 13.3(n=2), D N M T 3 A$ at $2 \mathrm{p} 23.3$ $(n=1), E Z H 2$ at $7 \mathrm{q} 36.1(n=1), F B X W 7$ at $4 \mathrm{q} 31.3(n=$ 12; one case harbored two different mutations), JAK1 at $1 \mathrm{p} 31.3(n=1), J A K 3$ at $19 \mathrm{p} 13.11(n=1)$, NOTCH1 at 9q34.3 ( $n=19$; two cases carried two and three different mutations, respectively), NRAS at $1 \mathrm{p} 13.2(n=2)$, PHF6 at Xq26.2 $(n=1)$, PIK3CA at $3 \mathrm{q} 26.32(n=1)$, PTEN at 10q23.31 $(n=2)$, SETD2 at 3p21.31 $(n=1)$, and TCF3 at 19 p13.3 $(n=1)$. Of the 19 NOTCH1 mutations, $17(89 \%)$ occurred in exons 26-27 (HD domain) and two (11\%) in exon 34 (PEST domain). Identical mutations were found in three genes: FBXW7 (c.1393C $>\mathrm{T}$ in four cases and c. $1513 \mathrm{C}>\mathrm{T}$ in three cases), NOTCH1 (c.4793G $>\mathrm{C}$ in two cases and c.5033 $\mathrm{T}>\mathrm{C}$ in three cases), and NRAS (c.34G > A in two cases). 


\section{Genetic comparisons between diagnostic and relapse samples}

Nine of the 47 patients relapsed, and samples from six of these were analyzed cytogenetically at both diagnosis and relapse (Figure 2 and Additional file 4: Table S4). At relapse, three cases $(\# 4,15$, and 21$)$ had additional aberrations. In one case (\#29), the diagnostic aberration was not seen at relapse, whereas two (\#6 and 10) displayed identical changes. FISH data on TCR rearrangements were available for five of the paired samples. In four of these, the FISH findings did not differ between diagnosis and relapse. In one case (\#15), the diagnostic sample was positive for a TRA/D rearrangement, whereas the relapses were negative.

SNP array analyses were performed on 11 paired samples, comprising seven $(\# 4,6,10,15,21,35$, and 47; Additional file 4: Table S4) in-house and four (\#U1-U4) external cases, with informative results being obtained in eight. In two cases (\#35 and U4), the aberrations at diagnosis and relapse were identical. In three cases (\#15, 21, and U1), all diagnostic abnormalities were present at relapse together with additional changes (clonal evolution). In three cases (\#6, 47, and U2), the diagnostic and relapse samples not only shared some aberrations but also harbored distinct aberrations (evolution from a preleukemic/ancestral clone). A total of 13 aberrations were seen only at relapse; none of these was recurrent. Targeted sequencing analyses were performed on three paired samples (cases 10, 15, and 47). In two of these (\#10 and 15), the results were concordant between diagnosis and relapse. In contrast, case 47 harbored a NOTCH1 mutation at diagnosis with a variant allele frequency (VAF) of 0.14 (Additional file 3: Table S3); this mutation was not detected at relapse.

\section{Survival analyses}

The probabilities of relapse-free survival (pRFS), eventfree survival (pEFS), and overall survival (pOS) did not differ with regard to age, white blood cell (WBC)

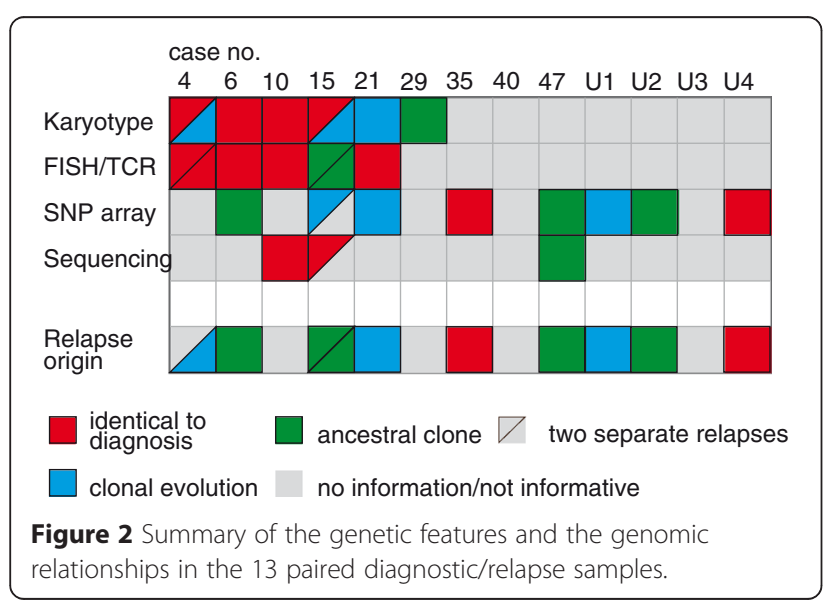

counts, and presence/absence of TCR translocations, STIL deletions, NOTCH1 and/or FBXW7 mutations, or mutations/deletions of genes involved in epigenetic regulation or signaling transduction. The pEFS and pOS (but not pRFS) were significantly lower in girls than in boys; however, this analysis was based on few cases (Additional file 5: Table S5).

The presence of $C D K N 2 A$ deletions was significantly associated with a high WBC count (median $209 \times 10^{9} / 1$ vs. $62 \times 10^{9} / \mathrm{l} ; P=0.013$ ). The pEFS and pRFS did not differ in relation to $C D K N 2 A$ status, whereas pOS was significantly lower $(P=0.04)$ for cases with $C D K N 2 A$ deletions (Additional file 5: Table S5). Among the 29 cases with loss of $C D K N 2 A$, there were no significant differences between those with $(n=12)$ and without sUPID9p $(n=17)$ as regards age (median 10.9 years vs. 7.0 years; $P=0.18)$, gender $(P=0.7)$, or WBC count (median $242 \times 10^{9} / 1$ vs. $\left.187 \times 10^{9} / 1 ; P=0.25\right)$.

\section{Discussion}

The rationale for the present study, representing a truly population-based pediatric T-ALL series comprising all 47 cases diagnosed in our catchment area between 1983 and 2011, was to ascertain whether a combination of SNP array and large-scale gene mutation analyses could add pathogenetically pertinent data, in particular as regards cooperative events. Taking the findings from all these investigatory modalities into account, it is apparent that T-ALL is characterized by multiple genetic changes (Figure 3), in line with a multistep leukemogenic process [5].

Previous studies using bacterial artificial chromosome or oligonucleotide arrays have identified recurrent deletions of several genes in T-ALL, such as BCL11B (14q32.2), EZH2 (7q36.1), LEF1 (4q25), NF1 (17q11.2), PHF6 (Xq26.2), PTEN (10q23.31), and PTPN2 (18p11.21) [6-12]. Although such investigations have yielded pathogenetically important information, the drawback of the abovementioned techniques is that they do not provide data on UPIDs. However, surprisingly few SNP array studies of pediatric T-ALL have been reported [5,13-18], and they have generally not discussed the frequency and distribution of UPIDs. Apart from detecting a median of three CNAs, mainly deletions, per case, similar to other studies $[13,17]$, and identifying microdeletions of CDKN2A, CDKN2B, LEF1, PHF6, PTEN, RB1, and STIL, we also observed a total of 18 sUPIDs targeting 6q, 9p, 10q, 13q, 17q, and 19p (Figure 1 and Additional file 1: Table S1). Interestingly, no wUPIDs were detected. In fact, when reviewing previous SNP array studies of pediatric T-ALL with data on UPIDs $[13,16,17]$, only one out of a total of 84 cases had a wUPID at diagnosis. When adding the present 40 cases, this would translate into a frequency of $0.8 \%(1 / 124)$ of wUPIDs in T-ALL. The reason for the 


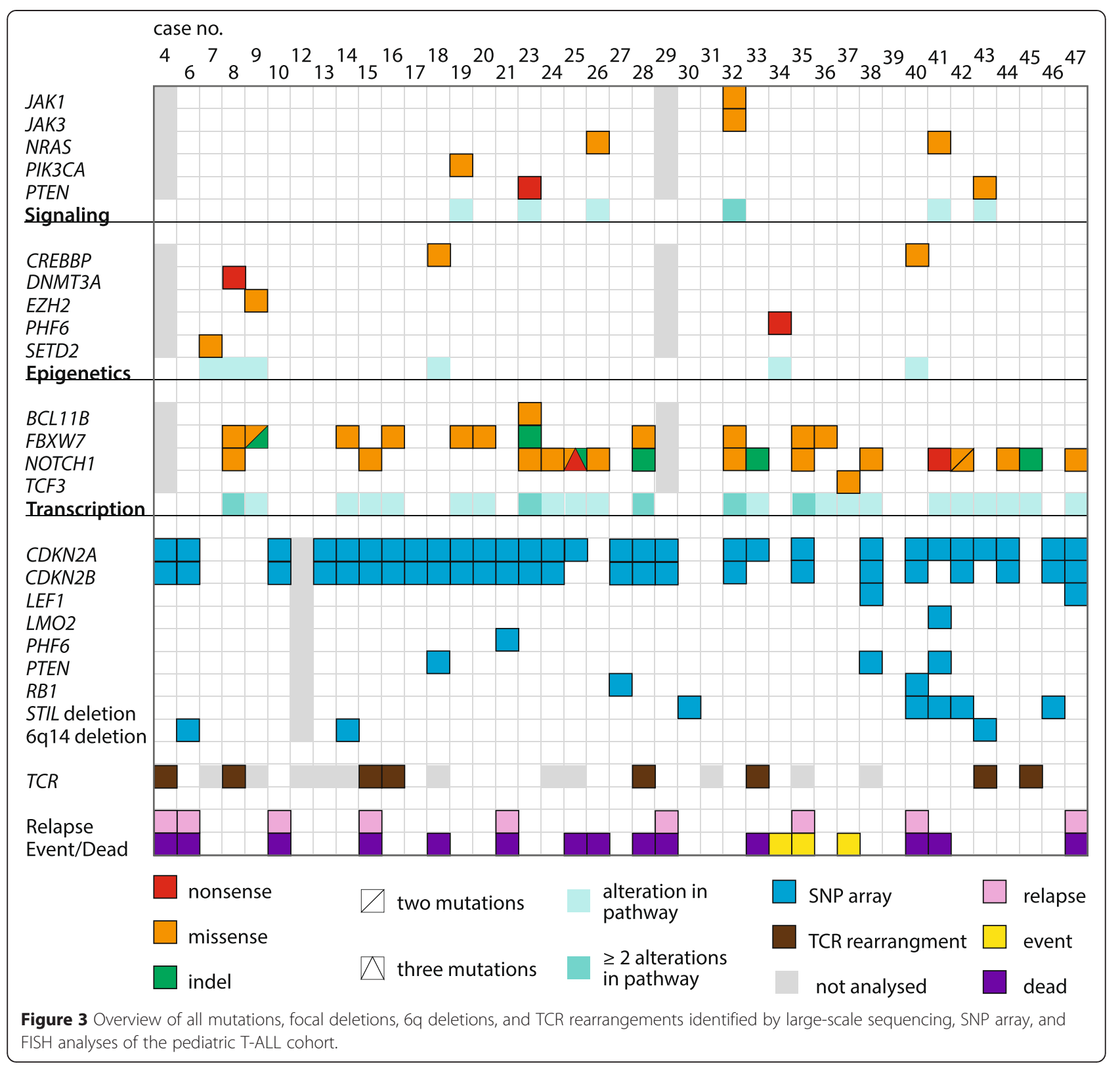

apparent lack of wUPIDs in T-ALL is unclear, but may simply be due to the fact that the vast majority of T-ALLs are pseudodiploid. Indeed, wUPIDs appear to be particularly common in aneuploid malignancies, such as hyperhaploid inflammatory leiomyosarcoma [19] and near haploid/ low hypodiploid B-cell precursor ALL [20] with chromosome doubling as well as in high hyperdiploid BCP ALL $[21,22]$. In these cases, the wUPIDs in most instances reflect the underlying mechanism of formation.

Except for sUPID9p, the other sUPIDs, involving 6q, $10 q, 13 q, 17 q$, and 19p, were non-recurrent. However, sUPIDs affecting $10 q$ and $17 q$, overlapping the sUPIDs identified in our study, have been reported in T-ALL $[13,16]$. The prior case with sUPID10q displayed a focal homozygous PTEN (10q23.31) deletion [13], suggesting that this could be the pathogenetically important outcome. In our case (\#10; Additional file 1: Table S1), PTEN was neither deleted nor mutated. As regards sUPID17q, the gene(s) possibly involved remains to be identified. In contrast, the target of sUPID9p, detected in $30 \%$ of the present T-ALL cases, is clearly CDKN2A at $9 \mathrm{p} 21.3$. This gene was deleted in $72 \%$ of all cases, a frequency similar to previous studies $[4,23,24]$. In $86 \%$ of these, the neighboring $C D K N 2 B$ gene was also deleted. Interestingly, $C D K N 2 A$ was homozygously deleted in all 12 cases with sUPID9p, whereas $C D K N 2 B$ was unaffected in $33 \%$ of these cases. Notably, all homozygous CDKN2A deletions associated with sUPID9p had identical breakpoints, 
strongly suggesting that a heterozygous deletion occurred prior to the sUPID.

In one of the investigated cases (U1), the SNP array analysis revealed a complex rearrangement of 6q, with a total of 28 breakpoints between $6 \mathrm{q} 14$ and 6qter and with copy number changes oscillating between one and two copies (Additional file 6: Figure SI), representing chromothripsis [25]. This phenomenon has been described in numerous tumor types [26], including a few cases of early T-cell precursor ALL [5]. Notably, the 6q abnormality in case $\mathrm{U} 1$ also harbored a larger proximal deletion, involving, among others, the CASP8AP2 gene at 6q15. This gene has been implicated as a prognostic marker in T-ALL $[7,27]$ and was deleted in all our TALL cases with del(6q).

Most previous mutation analyses of T-ALL have focused on only one or a few candidate genes, for example ETV6 (12p13.2), FBXW7 (4q31.3), FLT3 (13q12.2), IL7R (5p13.2), IRS4 (Xq22.3), JAK1 (1p31.3), NOTCH1 (9q34.3), NRAS (1p13.2), and TP53 (17p13.1). Although such an approach has been fruitful, it does not provide data on cooperative mutations in leukemogenesis [3,28-34]. More recently, studies applying whole genome or exome sequencing have been forthcoming, reporting an average of approximately ten protein altering mutations per pediatric T-ALL case $[5,35,36]$. In this study, we established a gene panel comprising 75 genes previously reported to be mutated in $\mathrm{T}$ ALL or shown to encode factors of importance in hematopoiesis. Using this approach, we obtained a mean sequencing depth of between 268 times and 465 times per sample, enabling detection of very small subclones.

Of the 39 samples sequenced, $28(72 \%)$ had one or several mutations, and of the 75 genes, 14 (19\%) were found to be mutated at least once. The genes targeted can broadly be categorized into one of the three groups: signaling transduction (JAK1, JAK3, NRAS, PI3KCA, and $P T E N$ mutations were seen in $15 \%$ of cases), epigenetic regulation (CREBBP, DNMT3A, EZH2, PHF6, and SETD2; 15\%), and transcription (BCL11B, FBXW7, NOTCH1, and TCF3; 59\%) (Figure 3). More than $75 \%$ of the mutations were present in major clones, as ascertained by VAFs above 0.25 (Additional file 3: Table S3). Because mutations may be misinterpreted as subclonal due to admixture of non-neoplastic cells, we only considered mutations with a VAF $<0.25$ to represent minor subclones if additional data showed a high proportion of malignant cells, i.e., if co-occurring mutations or CNAs/ sUPIDs were detected at higher frequencies. Using these criteria, subclones with FBXW7, NOTCH1, and/or PTEN were identified in six cases (Additional file 3: Table S3). NOTCH1 has been suggested to act as an initiating event in T-ALL $[37,38]$. However, the present findings, together with previous studies reporting low-level mutation frequencies of NOTCH1 and relapses of NOTCH1- positive T-ALL being negative for the mutation, as also seen in our case 47 (Additional file 4: Table S4) [39,40], show that NOTCH1 mutations also can be secondary events.

By combining the cytogenetic, FISH, SNP array, and mutation results in paired diagnostic and relapse samples, three different evolution patterns emerged: i) identical clones, ii) clonal evolution, and iii) ancestral clones (Figure 2 and Additional file 4: Table S4), in line with prior studies $[23,36,40]$. In no instance did we have any evidence for completely distinct clones at relapse. Thus, none of the relapses could be considered a "second" leukemia, as has been suggested in a few instances of T-ALL relapses [16]. However, the latter study focused on late relapses, i.e., those occurring more than 2.5 years after diagnosis, and most of our cases were early relapses. Quite few additional genetic changes were present at relapse (Additional file 4: Table S4), confirming that genomic instability is not a major feature of T-ALL [15].

\section{Conclusion}

The salient findings in the present study were the lack of wUPIDs, a high proportion of sUPID9p targeting CDKN2A, NOTCH1 mutations in subclones, and recurrent mutations of genes involved in signaling transduction, epigenetic regulation, and transcription in T-ALL.

\section{Methods}

\section{Patients and cytogenetic/FISH analyses}

Between 1983 and 2011, 47 children/adolescents (<18 years) were diagnosed with T-ALL in southern Sweden (Departments of Pediatric Hematology and Oncology, Lund and Linköping University Hospitals; Additional file 1: Table S1). The T-ALL diagnosis was based on immunophenotypic feature [41]. The male/female ratio was 4.2 , median age 9.1 years (range $0.7-17.2$ years), and the median WBC count was $92 \times 10^{9} / 1$ (range $\left.1.4-768 \times 10^{9} / \mathrm{l}\right)$. Mediastinal and central nervous system involvement was seen in $62 \%$ and $13 \%$ of informative cases, respectively (Additional file 7: Table S6). The pRFS, pEFS, and pOS at both 5 and 10 years were $0.815(0.06), 0.604$ (SE 0.07), and 0.665 (SE 0.07 ), respectively. The basic clinical features did not differ significantly (chi-square test) from those observed in a population-based cohort of 285 pediatric Nordic T-ALL patients previously reported by us [42]. Samples from 44 (94\%) of the patients were sent for cytogenetic analysis to the Department of Clinical Genetics, Lund University Hospital, Sweden. The median number of analyzed metaphases per case was 17 (range 0-49). All abnormal karyotypes have been centrally reviewed annually since 1996 by the Swedish Childhood Leukemia Cytogenetics Group, and five different treatment protocols were used during this time period by the Nordic Society for Pediatric Hematology and Oncology 
(NOPHO): NOPHO ALL-1981, -1986, -1992, -2000, and $-2008[43,44]$. The study was approved by the Regional Ethical Review Board at Lund University, and informed consent was obtained according to the Declaration of Helsinki.

In the present study, interphase FISH analyses using the TRA/TRD break apart FISH probe (Abbott, Stockholm, Sweden) and the TRB split signal FISH probe (Dako) were carried out on 31 diagnostic samples from which cells in fixative were available. Cutoff values (median $+3 \mathrm{SD}$ ) for TRA/TRD and TRB rearrangements, based on analyses of five normal bone marrow (BM) samples, were $4.9 \%$ and $1.1 \%$, respectively. TCR rearrangements, as ascertained by interphase FISH analysis, were identified in eight (26\%) of the 31 cases investigated; five involved TRA/TRD (\#8, 15, 16,28 , and 33) and three TRB (\#4,43, and 45). Six of these had been detected by G-banding analysis (\#8, 16, 28, 33, 43 , and 45), whereas two cases (4 and 15) had seemingly normal karyotypes (Additional file 1: Table S1). The frequency of FISH-identified TCR translocations (26\%), with some of them being cytogenetically cryptic, is in line with prior reports $[45,46]$.

\section{SNP array analysis-diagnostic samples}

DNA was available from 40 of the 44 samples and was extracted from BM $(n=33)$ or peripheral blood $(n=7)$; in $27 / 40(68 \%)$ cases, a remission sample could be included as a control. The HumanOmni1-Quad BeadChip, containing $>1$ million markers with a median marker spacing of $1.5 \mathrm{~kb}$ (Illumina, San Diego, CA, USA), was used. The analyses were performed according to the manufacturer's instructions, and data analysis was done using the GenomeStudio software 2011.1 (Illumina), extracting probe positions from the GRCh37 genome build (http://www.ensembl.org/Homo_sapiens/ Info/Index). Imbalances seen in remission samples or overlapping with copy number polymorphisms listed in the Database of Genomic Variants (http://projects. tcag.ca/variation/) were excluded from further analysis, and so were deletions involving the TCR and immunoglobulin loci because they most likely represent somatic rearrangements clonotypic for the malignant lymphoid cells rather than oncogenic events [47].

\section{SNP array analysis—paired diagnostic/relapse samples}

Of the 47 patients with T-ALL, nine relapsed (Additional file 7: Table S6). SNP array analyses could be performed on diagnostic as well as on relapse samples from seven of these (Additional file 1: Table S1 and Additional file 4: Table S4). These samples were analyzed using the Illumina platform described above. In addition, four paired diagnostic/relapse T-ALL (cases U1-U4) samples from the Department of Pediatric Hematology and Oncology, Norrland University Hospital, Umeå, Sweden, were available for analysis; these were investigated using the Illumina Humancnv370-Duo BeadChip containing $>370,000$ markers, with a median marker spacing of $4.9 \mathrm{~kb}$ (Illumina). The analyses were performed as above with the exception that the probe positions were extracted from the NCBI36.1 genome build.

\section{Targeted deep sequencing of 75 genes}

A sufficient amount of DNA for large-scale sequencing was available from 39 diagnostic and three relapse samples (Additional file 1: Table S1). The DNA quality was evaluated using the NanoDrop 1000 Spectrophotometer (Thermo Scientific, Wilmington, DE, USA) and by visual inspection of gel images of total genomic DNA. Seventyfive genes, comprising genes previously reported to be mutated in T-ALL or to participate in various cellular processes in T-cells, such as signaling transduction, epigenetic regulation, transcription, cell cycle control, cell-cell interaction, and DNA repair (Additional file 8: Table S7), were targeted for capture and deep sequencing. Using the eArray system (Agilent Technologies, Santa Clara, CA, USA), a SureSelect capture library was designed to include all exons and flanking intronic sequences. In addition, $5{ }^{\prime} \mathrm{UTR}$ and 3'UTR were added to the selected regions. A total of 15343 baits, which covered 95\% of the target bases, corresponded to a final capture size of $0.5 \mathrm{Mb}$. After DNA fragmentation, which yielded fragments of a median size of $251 \mathrm{bp}$, sequencing libraries were generated using the SureSelect post capture protocol for Illumina paired-end sequencing (Agilent). The samples were sequenced on an Illumina HiSeq 2000 platform using 100-bp paired-end reads. The sequences were aligned to the human genome reference hg 19 (http://hgdownload.cse.ucsc.edu/downloads. html\#human), with the short read alignment program Burrows-Wheeler Alignment tool [48]. Library preparation, sequencing, and alignment were performed by Science for Life Laboratory (SciLifeLab, Solna, Sweden). After alignment, each sample was evaluated by performing filtering and variant calling using the Genespring GX software (Agilent), including removal of duplicates, a confident score cutoff of 10 , base quality of 30 , and ignorance of reference locations under 5/1.

Only variants classified as non-synonymous and not present as germline SNPs in the dbSNP database were retained. Of the remaining variants, those fulfilling the following criteria were considered pathogenetically relevant: i) known cancer-associated mutations listed in the Ensembl genome browser (http://www.ensembl.org/ index.html) and ii) novel variants that could be confirmed by Sanger sequencing of diagnostic samples and that were not present in remission samples. Sanger dideoxy terminator sequencing was performed with primers designed with Primer3web (http://bioinfo.ut.ee/primer3/); primer sequences are available upon request. PCR was done using 
the BigDye ${ }^{\circ}$ Terminator v1.1 Cycle Sequencing Kit (Applied Biosystems, Foster City, CA, USA), and the sequencing products were resolved on the 3130 Genetic Analyzer (Applied Biosystems).

\section{Statistical analyses}

The IBM SPSS Statistics for Windows, Version 22 (Armonk, IBM Corp, NY, USA) was used for all statistical analyses. The significance limit for two-sided $P$ values was set to $<0.05$. The Mann-Whitney $U$ test was used to investigate possible correlations between CDKN2A deletions and age and WBC count. Fisher exact probability and Mann-Whitney $U$ tests were used to compare the distribution of gender, age, and WBC counts between $C D K N 2 A$ deleted cases with and without sUPID9p. The pRFS, pEFS, and pOS were calculated using the Kaplan-Meier method, and subgroups were compared using the log-rank test. The following parameters were compared: clinical features (age, gender, and WBC count), TCR translocations, SNP array findings, i.e., number of aberrations (CNAs and sUPIDs combined), and presence/absence of CDKN2A and STIL deletions, NOTCH1 and/or FBXW7 mutations, and mutations/deletions of genes encoding proteins involved in epigenetic regulation or signaling transduction. In the analysis of pEFS, events comprised induction failure, relapse, second malignant neoplasm, and death in complete remission 1 . In the OS analyses, death of any cause was the endpoint. Patients in continuous complete remission 1 were followed up between 0 and 304 months (median 66 months). The date of the last follow-up was January 10, 2012.

\section{Additional files}

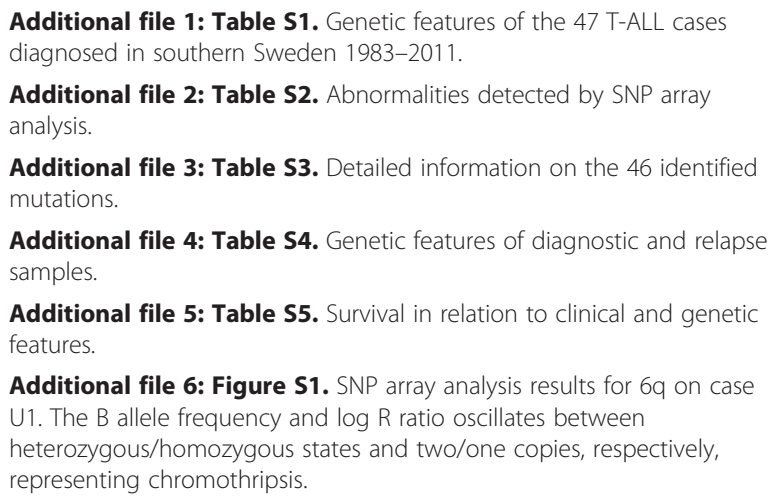

Additional file 4: Table S4. Genetic features of diagnostic and relapse samples.

Additional file 5: Table S5. Survival in relation to clinical and genetic features.

Additional file 6: Figure S1. SNP array analysis results for $6 \mathrm{q}$ on case U1. The B allele frequency and log $R$ ratio oscillates between heterozygous/homozygous states and two/one copies, respectively, representing chromothripsis.

Additional file 7: Table S6. Clinical and immunophenotypic features of the 47 T-ALL patients diagnosed in southern Sweden 1983-2011.

Additional file 8: Table S7. The 75 genes sequenced and their chromosomal positions.

\section{Competing interests}

The authors declare that they have no competing interests.

\section{Authors' contributions}

KK and BJ designed the study. KK, LO, AB, TF, and KP performed the molecular and/or data analyses. AC, MB, EF, and ME provided patient samples, clinical data, and/or immunophenotypic data. KK and BJ wrote the first draft of the manuscript. All authors contributed to write the manuscript and reviewed and approved the final version.

\section{Acknowledgements}

This study was supported by grants from the Swedish Cancer Society, the Swedish Childhood Cancer Foundation, and the Swedish Research Council. The Science for Life Laboratory (SciLifeLab in Stockhom), the national infrastructure SNISS, and Uppmax provided assistance in massive parallel sequencing and computational infrastructure.

\section{Author details}

'Department of Clinical Genetics, University and Regional Laboratories, Region Skåne, SE-221 85 Lund, Sweden. ²Division of Clinical Genetics, Department of Laboratory Medicine, Lund University, Lund, Sweden. ${ }^{3}$ Department of Pediatrics, Skåne University Hospital, Lund University, Lund, Sweden. ${ }^{4}$ Department of Pediatrics, Linköping University Hospital, Linköping, Sweden. ${ }^{5}$ Department of Medical Biosciences, Clinical Genetics, Umeå University, Umeå, Sweden. ${ }^{6}$ Department of Pathology, University and Regional Laboratories, Region Skåne, Lund, Sweden.

Received: 11 February 2015 Accepted: 13 April 2015

Published online: 24 April 2015

\section{References}

1. Kraszewska MD, Dawidowska M, Szczepański T, Witt M. T-cell acute lymphoblastic leukaemia: recent molecular biology findings. $\mathrm{Br} J$ Haematol. 2011;156:303-15.

2. Van Vlierberghe $P$, Pieters R, Beverloo HB, Meijerink JP. Molecular-genetic insights in paediatric T-cell acute lymphoblastic leukaemia. Br J Haematol. 2008;143:153-68.

3. Weng AP, Ferrando AA, Lee W, Morris JP, Silverman LB, Sanchez-lrizarry C, et al. Activating mutations of NOTCH1 in human T cell acute lymphoblastic leukemia. Science. 2004;306:269-71.

4. Yu L, Slovak ML, Mannoor K, Chen C, Hunger SP, Carroll AJ, et al. Microarray detection of multiple recurring submicroscopic chromosomal aberrations in pediatric T-cell acute lymphoblastic leukemia. Leukemia. 2011;25:1042-6.

5. Zhang J, Ding L, Holmfeldt L, Wu G, Heatley SL, Payne-Turner D, et al. The genetic basis of early T-cell precursor acute lymphoblastic leukaemia. Nature. 2012;481:157-63.

6. Balgobind BV, Van Vlierberghe $P$, van den Ouweland AM, Beverloo HB, Terlouw-Kromosoeto JN, van Wering ER, et al. Leukemia-associated NF1 inactivation in patients with pediatric T-ALL and AML lacking evidence for neurofibromatosis. Blood. 2008;111:4322-8.

7. Remke M, Pfister S, Kox C, Toedt G, Becker N, Benner A, et al. High-resolution genomic profiling of childhood T-ALL reveals frequent copy-number alterations affecting the TGF-beta and PI3K-AKT pathways and deletions at 6q15-16.1 as a genomic marker for unfavorable early treatment response. Blood. 2009;114:1053-62.

8. Gutierrez A, Sanda T, Ma W, Zhang J, Grebliunaite R, Dahlberg S, et al. Inactivation of LEF1 in T-cell acute lymphoblastic leukemia. Blood. 2010;115:2845-51.

9. Kleppe M, Lahortiga I, El Chaar T, De Keersmaecker K, Mentens N, Graux C, et al. Deletion of the protein tyrosine phosphatase gene PTPN2 in T-cell acute lymphoblastic leukemia. Nat Genet. 2010;42:530-5.

10. Van Vlierberghe P, Palomero T, Khiabanian H, Van der Meulen J, Castillo M, Van Roy N, et al. PHF6 mutations in T-cell acute lymphoblastic leukemia. Nat Genet. 2010;42:338-42.

11. Ntziachristos $P$, Tsirigos A, Van Vlierberghe P, Nedjic J, Trimarchi T, Flaherty MS, et al. Genetic inactivation of the polycomb repressive complex 2 in $T$ cell acute lymphoblastic leukemia. Nat Med. 2012;18:298-301.

12. Gutierrez A, Kentsis A, Sanda T, Holmfeldt L, Chen SC, Zhang J, et al. The $B C L 11 B$ tumor suppressor is mutated across the major molecular subtypes of T-cell acute lymphoblastic leukemia. Blood. 2011;118:4169-73.

13. Mullighan CG, Goorha S, Radtke I, Miller CB, Coustan-Smith E, Dalton JD, et al. Genome-wide analysis of genetic alterations in acute lymphoblastic leukaemia. Nature. 2007;446:758-64. 
14. Kawamata N, Ogawa S, Zimmermann M, Kato M, Sanada M, Hemminki K, et al. Molecular allelokaryotyping of pediatric acute lymphoblastic leukemias by high-resolution single nucleotide polymorphism oligonucleotide genomic microarray. Blood. 2008;111:776-84

15. Tosello V, Mansour MR, Barnes K, Paganin M, Sulis ML, Jenkinson S, et al. WT1 mutations in T-ALL. Blood. 2009;114:1038-45.

16. Szczepański T, van der Velden VH, Waanders $E$, Kuiper RP, Van Vlierberghe $P$, Gruhn B, et al. Late recurrence of childhood T-cell acute lymphoblastic leukemia frequently represents a second leukemia rather than a relapse: first evidence for genetic predisposition. J Clin Oncol. 2011;29:1643-9.

17. La Starza R, Lettieri A, Pierini V, Nofrini V, Gorello P, Songia S, et al. Linking genomic lesions with minimal residual disease improves prognostic stratification in children with T-cell acute lymphoblastic leukaemia. Leuk Res. 2013:37:928-35.

18. Olsson L, Castor A, Behrendtz M, Biloglav A, Forestier E, Paulsson K, et al. Deletions of IKZFI and SPREDI are associated with poor prognosis in a population-based series of pediatric B-cell precursor acute lymphoblastic leukemia diagnosed between 1992 and 2011. Leukemia. 2014;28:302-10.

19. Nord KH, Paulsson K, Veerla S, Wejde J, Brosjö O, Mandahl N, et al. Retained heterodisomy is associated with high gene expression in hyperhaploid inflammatory leiomyosarcoma. Neoplasia. 2012;14:807-12.

20. Safavi S, Forestier E, Golovleva I, Barbany G, Nord KH, Moorman AV, et al. Loss of chromosomes is the primary event in near-haploid and low-hypodiploid acute lymphoblastic leukemia. Leukemia. 2013;27:248-50.

21. Paulsson $\mathrm{K}$, Johansson B. High hyperdiploid childhood acute lymphoblastic leukemia. Genes Chromosomes Cancer. 2009;48:637-60.

22. Paulsson K, Forestier E, Lilljebjörn H, Heldrup J, Behrendtz M, Young BD, et al. Genetic landscape of high hyperdiploid childhood acute lymphoblastic leukemia. Proc Natl Acad Sci U S A. 2010;107:21719-24.

23. Mullighan CG, Phillips LA, Su X, Ma J, Miller CB, Shurtleff SA, et al. Genomic analysis of the clonal origins of relapsed acute lymphoblastic leukemia. Science. 2008;322:1377-80.

24. Sulong S, Moorman AV, Irving JA, Strefford JC, Konn ZJ, Case MC, et al. A comprehensive analysis of the CDKN2A gene in childhood acute lymphoblastic leukemia reveals genomic deletion, copy number neutral loss of heterozygosity, and association with specific cytogenetic subgroups. Blood. 2009;113:100-7.

25. Stephens PJ, Greenman CD, Fu B, Yang F, Bignell GR, Mudie LJ, et al Massive genomic rearrangement acquired in a single catastrophic event during cancer development. Cell. 2011;144:27-40.

26. Cai H, Kumar N, Bagheri HC, von Mering C, Robinson MD, Baudis M. Chromothripsis-like patterns are recurring but heterogeneously distributed features in a survey of 22,347 cancer genome screens. BMC Genomics. 2014;15:1-29.

27. Flotho C, Coustan-Smith E, Pei D, Iwamoto S, Song G, Cheng C, et al. Genes contributing to minimal residual disease in childhood acute lymphoblastic leukemia: prognostic significance of CASP8AP2. Blood. 2006;108:1050-7.

28. Kawamura M, Ohnishi H, Guo SX, Sheng XM, Minegishi M, Hanada R, et al. Alterations of the $p 53, p 21, p 16, p 15$ and RAS genes in childhood T-cell acute lymphoblastic leukemia. Leuk Res. 1999;23:115-26.

29. Van Vlierberghe P, Meijerink JP, Stam RW, van der Smissen W, van Wering ER, Beverloo HB, et al. Activating FLT3 mutations in CD4+/CD8- pediatric T-cell acute lymphoblastic leukemias. Blood. 2005;106:4414-5.

30. Van Vlierberghe P, Ambesi-Impiombato A, Perez-Garcia A, Haydu JE, Rigo I, Hadler $M$, et al. ETV6 mutations in early immature human T cell leukemias. J Exp Med. 2011;208:2571-9.

31. Malyukova A, Dohda T, von der Lehr N, Akhoondi S, Corcoran M, Heyman $\mathrm{M}$, et al. The tumor suppressor gene hCDC4 is frequently mutated in human T-cell acute lymphoblastic leukemia with functional consequences for Notch signaling. Cancer Res. 2007;67:5611-6.

32. Flex E, Petrangeli V, Stella L, Chiaretti S, Hornakova T, Knoops L, et al. Somatically acquired JAK1 mutations in adult acute lymphoblastic leukemia. J Exp Med. 2008;205:751-8

33. Karrman K, Isaksson M, Paulsson K, Johansson B. The insulin receptor substrate 4 gene (IRS4) is mutated in paediatric T-cell acute lymphoblastic leukaemia. Br J Haematol. 2011:155:516-9.

34. Zenatti PP, Ribeiro D, Li W, Zuurbier L, Silva MC, Paganin M, et al. Oncogenic IL7R gain-of-function mutations in childhood T-cell acute lymphoblastic leukemia. Nat Genet. 2011;43:932-9.
35. De Keersmaecker K, Atak ZK, Li N, Vicente C, Patchett S, Girardi T, et al. Exome sequencing identifies mutation in CNOT3 and ribosomal genes RPL5 and RPL10 in T-cell acute lymphoblastic leukemia. Nat Genet. 2013;45:186-90.

36. Tzoneva G, Perez-Garcia A, Carpenter Z, Khiabanian H, Tosello V, Allegretta M, et al. Activating mutations in the NT5C2 nucleotidase gene drive chemotherapy resistance in relapsed ALL. Nat Med. 2013;19:368-71.

37. Blackburn JS, Liu S, Raiser DM, Martinez SA, Feng H, Meeker ND, et al. Notch signaling expands a pre-malignant pool of T-cell acute lymphoblastic leukemia clones without affecting leukemia-propagating cell frequency. Leukemia. 2012;26:2069-78.

38. Armstrong F, Brunet de la Grange P, Gerby B, Rouyez MC, Calvo J, Fontenay $\mathrm{M}$, et al. NOTCH is a key regulator of human T-cell acute leukemia initiating cell activity. Blood. 2009;113:1730-40.

39. Mansour MR, Duke V, Foroni L, Patel B, Allen CG, Ancliff PJ, et al. Notch-1 mutations are secondary events in some patients with T-cell acute lymphoblastic leukemia. Clin Cancer Res. 2007;13:6964-9.

40. Clappier E, Gerby B, Sigaux F, Delord M, Touzri F, Hernandez L, et al. Clonal selection in xenografted human $T$ cell acute lymphoblastic leukemia recapitulates gain of malignancy at relapse. J Exp Med. 2011;208:653-61.

41. Bene MC, Castoldi G, Knapp W, Ludwig WD, Matutes E, Orfao A, et al. Proposals for the immunological classification of acute leukemias. European Group for the Immunological Characterization of Leukemias (EGIL). Leukemia. 1995;9:1783-6.

42. Karrman K, Forestier E, Heyman M, Andersen MK, Autio K, Blennow E, et al. Clinical and cytogenetic features of a population-based consecutive series of 285 pediatric T-cell acute lymphoblastic leukemias: rare T-cell receptor gene rearrangements are associated with poor outcome. Genes Chromosomes Cancer. 2009;48:795-805.

43. Gustafsson G, Schmiegelow K, Forestier E, Clausen N, Glomstein A, Jonmundsson $\mathrm{G}$, et al. Improving outcome through two decades in childhood ALL in the Nordic countries: the impact of high-dose methotrexate in the reduction of CNS irradiation. Leukemia. 2000;14:2267-75.

44. Schmiegelow K, Forestier E, Hellebostad M, Heyman M, Kristinsson J, Söderhäll S, et al. Long-term results of NOPHO ALL-92 and ALL-2000 studies of childhood acute lymphoblastic leukemia. Leukemia. 2010;24:345-54.

45. Cauwelier B, Dastugue N, Cools J, Poppe B, Herens C, De Paepe A, et al. Molecular cytogenetic study of 126 unselected T-ALL cases reveals high incidence of TCR $\beta$ locus rearrangements and putative new T-cell oncogenes. Leukemia. 2006;20:1238-44.

46. Le Noir S, Ben Abdelali R, Lelorch M, Bergeron J, Sungalee S, Payet-Bornet $D$, et al. Extensive molecular mapping of TCRa/ $\delta$ - and TCR $\beta$-involved chromosomal translocations reveals distinct mechanisms of oncogene activation in T-ALL. Blood. 2012;120:3298-309.

47. Wlodarska I, Matthews C, Veyt E, Pospisilova H, Catherwood MA, Poulsen TS, et al. Telomeric $/ G H$ losses detectable by fluorescence in situ hybridization in chronic lymphocytic leukemia reflect somatic $V_{H}$ recombination events. J Mol Diagn. 2007;9:47-54.

48. Li H, Durbin R. Fast and accurate short read alignment with Burrows-Wheeler transform. Bioinformatics. 2009;25:1754-60.

\section{Submit your next manuscript to BioMed Central and take full advantage of:}

- Convenient online submission

- Thorough peer review

- No space constraints or color figure charges

- Immediate publication on acceptance

- Inclusion in PubMed, CAS, Scopus and Google Scholar

- Research which is freely available for redistribution 